\title{
Using Transfer Learning and Hierarchical Classifier to Diagnose Melanoma From Dermoscopic Images
}

\author{
Priti Bansal, Netaji Subhas University of Technology, New Delhi, India \\ Sumit Kumar, Amity University, Noida, India \\ Ritesh Srivastava, GCET, India \\ (iD https://orcid.org/0000-0003-0960-4575 \\ Saksham Agarwal, Netaji Subhas Institute of Technology, New Delhi, India
}

\begin{abstract}
The deadliest form of skin cancer is melanoma, and if detected in time, it is curable. Detection of melanoma using biopsy is a painful and time-consuming task. Alternate means are being used by medical experts to diagnose melanoma by extracting features from skin lesion images. Medical image diagnosis requires intelligent systems. Many intelligent systems based on image processing and machine learning have been proposed by researchers in the past to detect different kinds of diseases that are successfully used by healthcare organisations worldwide. Intelligent systems to detect melanoma from skin lesion images are also evolving with the aim of improving the accuracy of melanoma detection. Feature extraction plays a critical role. In this paper, a model is proposed in which features are extracted using convolutional neural network (CNN) with transfer learning and a hierarchical classifier consisting of random forest $(\mathrm{RF})$, k-nearest neighbor $(\mathrm{KNN})$, and adaboost is used to detect melanoma using the extracted features. Experimental results show the effectiveness of the proposed model.
\end{abstract}

\section{KEYWORDS}

Adaboost, Convolution Neural Network, Dermoscopic Images, Feature Extraction, Hierarchical Classifier, K-Nearest Neighbor (KNN), Melanoma, Random Forest (RF), Transfer Learning, VGG-16

\section{INTRODUCTION}

The increasingly growing amount of data in the field of healthcare industry world-wide necessitate the need of efficient data handling and analyzing techniques. Proper handling and analysis of data by intelligent machines can help in improving the disease detection (cardiovascular disease, cancer, etc.) capability as compared to manual techniques (Priyanga and Naveen, 2018). Skin cancer is nowa-days rampant in many parts of the world especially in the United States and Asian countries. There are three major types of skin cancers namely basal cell carcinoma (BBQ), squamous cell carcinoma (SCC) and melanoma, out of which melanoma is the most dangerous form of skin cancer. Melanoma develops when unrepairable damage is caused to the skin cells called melanocytes which produce the pigment melanin to protect us from the UV radiations. Melanoma is generally caused due to exposure to ultra violet (UV) radiations. Melanoma is characterized by the development of skin lesions that

\section{DOI: 10.4018/IJHISI.20210401.oa4}

This article, published as an Open Access article on December 4, 2020 in the gold Open Access journal, International Journal of Healthcare Information Systems and Informatics (IJHISI) (converted to gold Open Access January 1, 2021), is distributed under the terms of the Creative Commons Attribution License (http://creativecommons.org/licenses/by/4.0/) which permits unrestricted use, distribution, and production in any medium, provided the author of the original work and original publication source are properly credited. 
may vary in color, size, shape and texture. The color of the skin usually becomes black or brown but sometimes the color might also be red, blue, purple or white. In the United States, approximately 5.4 million new cases of skin cancer are detected every year. Despite the fact that only 5\% cases represent melanoma, $75 \%$ of the skin cancer related deaths are due to melanoma, which accounts for around 10,000 deaths annually (Esteva et al., 2017).

Early detection of melanoma increases the chances of its curability as well as the life expectancy of the patient. Earlier, detection of skin cancer required a manual procedure which involves skin biopsy that was both time consuming and expensive. To overcome this problem, alternate means are being used by medical experts to diagnose melanoma using images of skin lesions. Medical image diagnosis requires intelligent systems. Many intelligent systems based on image processing, pattern recognition and machine learning have been proposed by researchers in past where millions of images can be processed at a time and can be used to predict or classify the image as melanomic or non melanomic. Skin lesions are examined and analyzed using a non-invasive procedure known as dermoscopy. Although dermoscopy improved the accuracy of detection of melanoma and reduced the number of biopsies as compared to naked eye examination, however it requires extensive training and expertise. Moreover, dermoscopic images are not available readily. Another method adopted by researchers nowadays is the use of conventional camera to capture the image of the pigmented region of the skin. Images collected from various clinics across the world are also used by researchers to classify them as melanomic or non-melanomic. The images collected from conventional camera are called as non-dermoscopic images. As compared to dermoscopic image, non-dermoscopic image contains fewer details and may have illumination and noise problem but these are readily available (Jafari et al., 2017a). An example of dermoscopic and non-dermoscopic images of melanoma taken from ISIC website and Med-node dataset (Giotis et al., 2015) respectively are shown in Figure 1.

To classify the skin lesions properly, it is important to extract relevant features from the images which helps in identifying the image. Features play a critical role in processing of images, especially

Figure 1. a) Dermoscopic images of melanoma b) non-dermoscopic images of melanoma
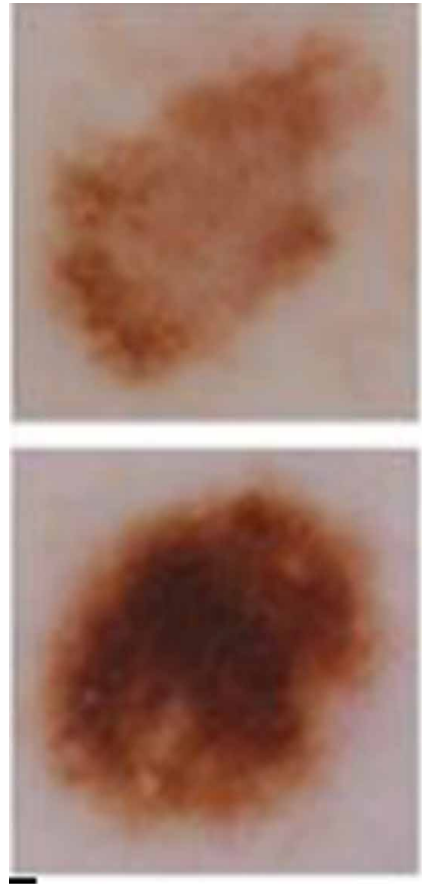
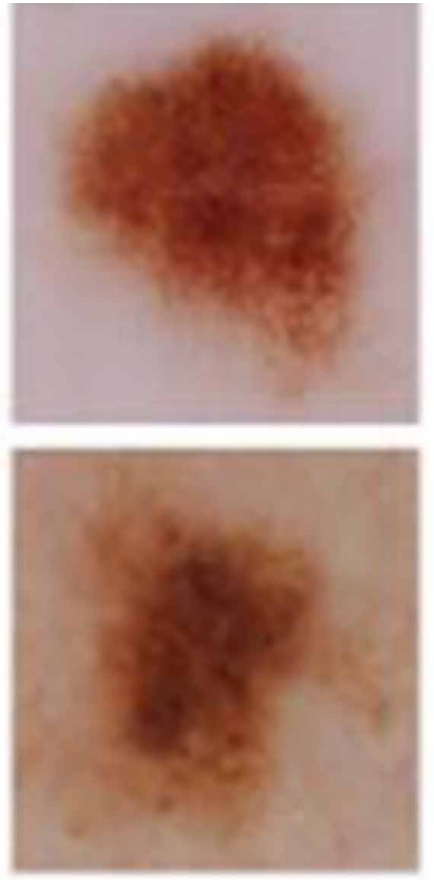

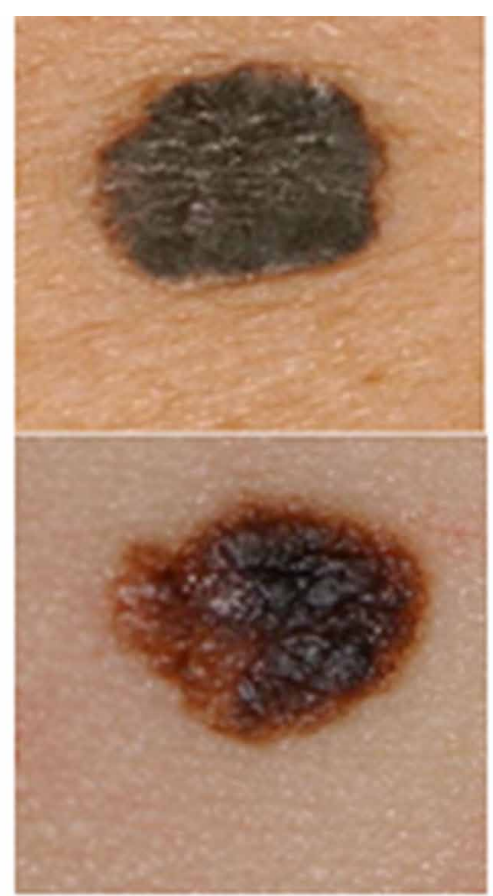


in the field of healthcare applications. This motivated the work presented in this paper. To classify an image, the first step is to extract features from the image. Features represent the information contained in the image that is used to classify the lesion. Features can be extracted using hand-crafted feature extraction techniques which can further be classified into global and local feature extraction techniques. Recently, convolution neural networks $(\mathrm{CNN})$ are also being used by researchers as a feature extractor. Once the features are extracted, the next step is to select relevant features from the extracted features that contribute in the classification of the image. A proper selection of features prevents underfitting and overfitting during the training process of the classifier and it helps in improving the accuracy of the classifier by removing noisy features (Puyalnithi and Vankadara, 2018). A lot of techniques exist in literature to extract features from dermoscopic as well as non-dermoscopic images using image processing techniques and to classify them as melonomic /non-melanomic image (Giotis et al., 2015; Jain et al., 2015; Jafari et al., 2017a; Jafari et al. 2017b; Kavitha et al., 2017). Recently, deep learning techniques have been explored by researchers to perform image segmentation and classification in the field of medical sciences (Melinscak et al., 2015; Havaei et al., 2016; Kalouche, 2016; Salido and Ruiz Jr., 2018; Seetharaman, 2019). In this paper, convolution neural networks (CNN) with transfer learning is used to extract feature from dermoscopic images and the extracted features are classified using a hierarchical classifier that consist of Random forest (RF), K-nearest neighbor (KNN) and Adaboost. The advantage of using CNN with transfer learning is two folds. Firstly, CNNs are good feature extractors. Secondly, by using transfer learning the training time of the CNN can be avoided. The main objective behind the use of hierarchical classifier is to ensure that the images classified by $\mathrm{RF}$ as non-melanomic are classified correctly. Therefore, KNN and Adaboost algorithms are used to verify the results of RF on negative cases.

The organization of the paper is as follows. Section 2 describes the related work. Section 3 gives a brief overview of CNN and various classifiers used in this paper. Section 4 presents the proposed work. Experimental setup and results are discussed in Section 5. Conclusion and Future work are discussed in Section 6.

\section{RELATED WORK}

Several methods exist in literature to detect melanoma from dermoscopic as well as non- dermoscopic images, where the features are extracted using either hand-crafted techniques or CNN. Celebi et al. (2007) proposed a method to classify dermoscopy images in which JSEG algorithm is used to detect the border automatically, Euclidean distance transform algorithm and GLCM are used to extract textural features and color histogram in six color spaces are used for color features extraction. After feature extraction, classification is done using SVM. In Mhaske \& Phalke (2013), both supervised (SVM and neural network) and unsupervised learning (K-means clustering) algorithms are used by authors to detect and classify melanoma. Two-dimensional wavelet method is used for extracting features which are given as input to the classifiers. Better results were given by the SVM in comparison to the other two algorithms. In Barata et al. (2014), skin lesions are segmented using simple adaptive thresholding algorithm. Then the textural features are extracted using GLCM in four different orientation angles. Color features are also extracted by color histograms in six color spaces. Finally, the extracted features are classified using SVM-RBF. It has been found during experimentation that the color features give better classification accuracy as compared to the textural features. Choudhury et.al (2015) proposed a multilayer decomposition aided method which uses GLCM and HOG (Histogram of Oriented Gradients) to extract textural and color features respectively from the images of skin cancer. These features are fed to Multiclass SVM and Extreme Learning Machine (ELM) to classify skin cancer cells into four classes namely Squamous cell carcinoma, Basal cell carcinoma, Melanoma and Actinic keratosis for DermNet NZ database. In Kavitha et al. (2017), skin lesion is extracted from the dermoscopic image using segmentation. It is done using Otsu's adaptive thresholding method and the global and local texture features extraction is performed using GLCM and SURF respectively. 
Finally, classification of melanoma is done using SVM and KNN. In Hardian et al. (2017), features are extracted using ABCD (A-Asymmetry, B-Border, C-Color and D-Diameter) rule from the preprocessed images. After feature extraction, a Total Dermoscopy Score (TDS) is calculated and the images are classified based on the value of TDS. The main drawback of this approach is that sometimes it may not recognize malignant moles at early stage. In Dubai et al. (2017), features are extracted from the segmented images using ABCD rule and classification is performed using neural network. However, as backpropagation is used to train neural network, the convergence speed of the proposed method is slow and there are chances of getting trapped in local minima.

In Giotis et al. (2015), a decision support system MED-NODE was developed to diagnose melanoma that utilizes image processing techniques to extracts the lesion regions from the nondermoscopic images. The extracted features along with the set of visual attributes provided by the physician are used for automatic prediction. Classification is done by a majority vote of all predictions. In Kalouche (2016), three models namely logistic regression, a deep neural network and a CNN with transfer learning are used to classify melanoma. First, the images are preprocessed and the skin lesions are segmented. The raw images are resized into equal size and are supplied as input to the various learning algorithms for classification. In Esteva et al. (2017), CNN was used to classify skin lesions. First the CNN was trained from clinical images directly where pixels and disease labels were given as input. The performance of $\mathrm{CNN}$ was compared against 21 board-certified dermatologists on biopsy-proven clinical images and the results shows the compatibility of artificial intelligence techniques with the expertise of dermatologist. In Anas et al. (2017), K-means clustering is used for image segmentation and then features are extracted, which are given as input to four different classifiers namely Nearest Neighborhood (NN), Nearest Mean Classifier (NMC), linear classifier, and Support Vector Classifier (SVC). In Dorj et al. (2018), a pretrained AlexNet CNN along with ECOC SVM is used for classifying skin cancer. Li and Shen (2018) proposed a deep learning model that consist of two fully connected residual networks (FCRN) to perform segmentation and classification simultaneously. Feature extraction is performed by straight-forward CNN. Salido and Ruiz Jr. (2018) use $\mathrm{CNN}$ to detect melanoma from dermoscopy images in $\mathrm{PH}^{2}$ dataset. First, images are preprocessed and hair are removed. After that feature extraction and classification is done using AlexNet. For good performance of a classifier, it is important that it should be trained using good quality training images. However, in the field of medical image processing, image enhancement and segmentation are difficult task. Dhal et al. (2018) presented a methodology to enhance the skin lesion images using hue-saturation-intensity (eHSI) color model and contrast limited histogram equalization (CLAHE). The enhanced images are segmented using unsupervised clustering approach. In Brinker et al. (2019a), a comparison is done between the performance of deep learning algorithm trained using open-source images with the performance of several dermatologists at different level of hierarchy and it has been shown that CNN is capable of performing better than dermatologists and can assist dermatologists to detect melanoma. The application of deep learning in melanoma detection is limited by availability of skin lesion images. The available datasets are highly imbalanced and contains images with occlusion. Bisla et al. (2019) built a deep learning tool for data purification and augmentation to counter-act these limitations. The data generation unit is based on generative adversarial networks. In Seetharaman (2019), a classifier based on multivariate parametric statistical tests of hypotheses is proposed which classifies skin images into normal and melanoma. It also further classifies melanoma images into two classes namely initial stage and advanced stage depending upon the extent of aggressiveness of the cancer by extracting textural, color and shape features. In Brinker et al. (2019b), a statistical test is performed that showed the superiority of deep learning network as compared to dermatologists in classifying melanoma images. Three McNemar's tests were performed to compare the results of the CNN's test runs with respect to sensitivity, specificity and overall correctness.

To analyze the performance of existing state-of-the-art techniques for melanoma image classification, the sensitivity, specificity and accuracy of the proposed classifiers are compared. 
The deep learning networks are first trained using the images in the training set. After training is performed, the images in the test dataset are classified and the results are compared to the existing state-of-the-art algorithms or to the performance of dermatologists.

\section{OVERVIEW}

This section presents a brief overview of the concepts used in this paper.

\section{Convolution Neural Network (CNN)}

CNN are deep neural networks primarily used to analyze images. The efficacy of CNNs in image recognition and the little pre-processing that is required as compared to other image classification algorithms are major advantages of $\mathrm{CNN}$ that has increased its usage in various domains such as image and video recognition, medical image analysis, robotics, object recognition etc. CNN architecture consists of three types of layers: Convolution Layer, Pooling Layer and Fully-Connected Layer. These layers are stacked together to form a full $\mathrm{CNN}$ architecture. CNN perceive images as 3-dimensional objects. So, it takes as input an image whose width and height are measured by the number of pixels along those dimensions, and depth is three layers deep in case of colored images and one layer deep for gray scale images. Those depth layers are referred to as channels. The image which is given as input to the CNN passes through a series of convolution layers with filters, pooling and fully connected layer. At the fully connected layer, a SoftMax activation function is applied to classify the input image.

\section{Transfer Learning}

Transfer learning is a technique where a model that has been trained for a particular task is exploited to be used to a new related task. It is very popular in deep learning. In transfer learning, a model trained on ImageNet dataset comprising of over 1.2 million images and over 1000 classes is used or is fine tuned. Some dense layers are added to this model at the end and these layers are trained with the actual dataset. The advantage of using pre-trained model is that it requires very less training as compared to training a CNN from scratch. The newly trained model can be used to predict the class of unseen images. Some of the existing pre-trained models are AlexNet, Inception, VGG-16, VGG-19 etc.

AlexNet (Krizhevsky et al.) is a CNN that is trained on more than a million images from the ImageNet dataset ${ }^{1}$. AlexNet consist of eight layers: five convolution and the remaining fully-connected layers. It uses Relu activation function which increases the speed without affecting the speed. It has 60 million parameters. The VGG network (Simonyan \& Zisserman, 2014) is characterized by its simplicity that uses only $3 \times 3$ convolutional layers stacked on top of each other in increasing depth. Max pooling is used to handle reducing volume size. As compared to AlexNet which has a receptive field of $11 \times 11$ with a stride of 4 , VGG uses very small receptive field of $3 \times 3$ with a stride of 1 . The small-sized convolution filters enable VGG to have more weight layers which in turn enhances its performance. The number of parameters in VGG are 138 million. VGG-16 and VGG-19 differ in the number of weight layers in the network. VGG is one of the most widely used architecture. The Inception architecture was first introduced by Szegedy et al. (2015). It was called GoogLeNet or Inception-v1. Since then various improvements of Inception architecture have been introduced. Inception-v1 is a 27-layer deep CNN. It consists of many inception modules connected together. The number of parameters in Inception-v1 is 6 million. In ILSVRC 2014 competition, Inception-v1 has achieved higher accuracy as compared to AlexNet and VGG.

\section{Support Vector Machine (SVM)}

SVM are supervised learning algorithms used for classification and regression problems. Each data point is plotted in $\mathrm{N}$-dimensional space. Then a hyperplane or a set of hyperplanes are constructed in $\mathrm{N}$-dimensional space to classify the training data. Classification is done by identifying the hyperplane 
having the highest margin to the nearest training data point of any class. Generally, the larger the margin, the lower is the generalization error.

\section{K-Nearest Neighbor (KNN)}

It is one of the most basic machine learning algorithms which is used for classification problems. A test data point is classified by finding out its ' $\mathrm{k}$ ' nearest neighbors ( $\mathrm{k}$ nearest training data point) and the class of majority of the k-nearest neighbors is predicted for that data point. Two important factors that need to be decided before classification is the value of ' $\mathrm{k}$ ' and the distance matrix. Some popular distance matrix used in KNN are Euclidean distance, Manhattan distances and Cosine similarity.

\section{Random Forest (RF)}

RF is an ensemble learning method for classification or regression task. Due to its simplicity, it is one of the most commonly used algorithms for classification. It builds a forest which is a collection of multiple decision trees. The model is trained by the 'bagging method'. The principle behind bagging method is that using a combination of learning algorithms will increase the overall efficiency based on the voting between the multiple decision trees.

\section{Adaboost}

Adaboost (Adaptive Boosting) is an ensemble method that attempts to create strong classifiers with the use of weak classifiers. Adaboost is successful especially in case of binary classification. First, a weak classifier is built from the training data. A misclassification rate is obtained using the training dataset. After this, a second model is built that attempts to correct the errors from the first model. The weak classifier used initially is the decision stump, which is generally a first level decision tree having one node. Then the pre-initialized weights are updated after a certain prediction is made. So, it is a technique which has the properties of back propagation and decision trees or ensemble methods and hence can be used on failed cases to further boost up the performance.

\section{Proposed Approach}

In this paper, a model is proposed to detect and classify melanoma from dermoscopic images. Before classification, it is important to extract useful features to improve the accuracy of classification. The proposed model uses VGG-16 (Simonyan \& Zisserman, 2014) a pre-trained 16-layer deep CNN trained on ImageNet dataset and developed by Visual Geometry Group (VGG) to extract features from the dermoscopic images. VGG-16 model consists of 13 convolution layers and 3 fully connected layers with SoftMax function at the final layer. VGG-16 uses a receptive field of size $3 \times 3$ and max- pooling layer of size $2 \times 2$. The size of images given as input to VGG-16 is $224 \times 224 \times 3$ and it classify them into 1000 classes. When the fully connected layers are removed from the VGG-16 model, it acts as a feature extractor for the input images and produces a feature vector (Gopalakrishnan et al., 2017). These image feature vectors can then be used to train and test any classifier.

In the proposed work, dermoscopic images dataset is taken from ISIC (International Skin Imaging Collaboration) website. The dataset is divided into two parts: training dataset and testing dataset. As input to VGG-16 is of size $224 \times 224 \times 3$, a preprocessing step is applied to resize the dermoscopic images to the desired size. The images are given as input to VGG-16 which convert them into 512-dimensional feature vector thereby enabling the extraction of features. The extracted feature vectors of the training dataset are given as input to a hierarchical classifier. The hierarchical classifier is trained using the extracted features. The hierarchical classifier consists of random forest, $\mathrm{KNN}$ and Adaboost. Initially, random forest is used for the classification of the image and if an image is classified as non-melanomic, then it is again tested using KNN and Adaboost at second level in order to double check the claim. At the second level of our hierarchical classification model, the decisions of KNN and Adaboost are integrated using an integration rule to generate the final decision. Majority voting is used for decision integration. The majority voting algorithm is used to construct an 
Figure 2. Block diagram of the proposed approach

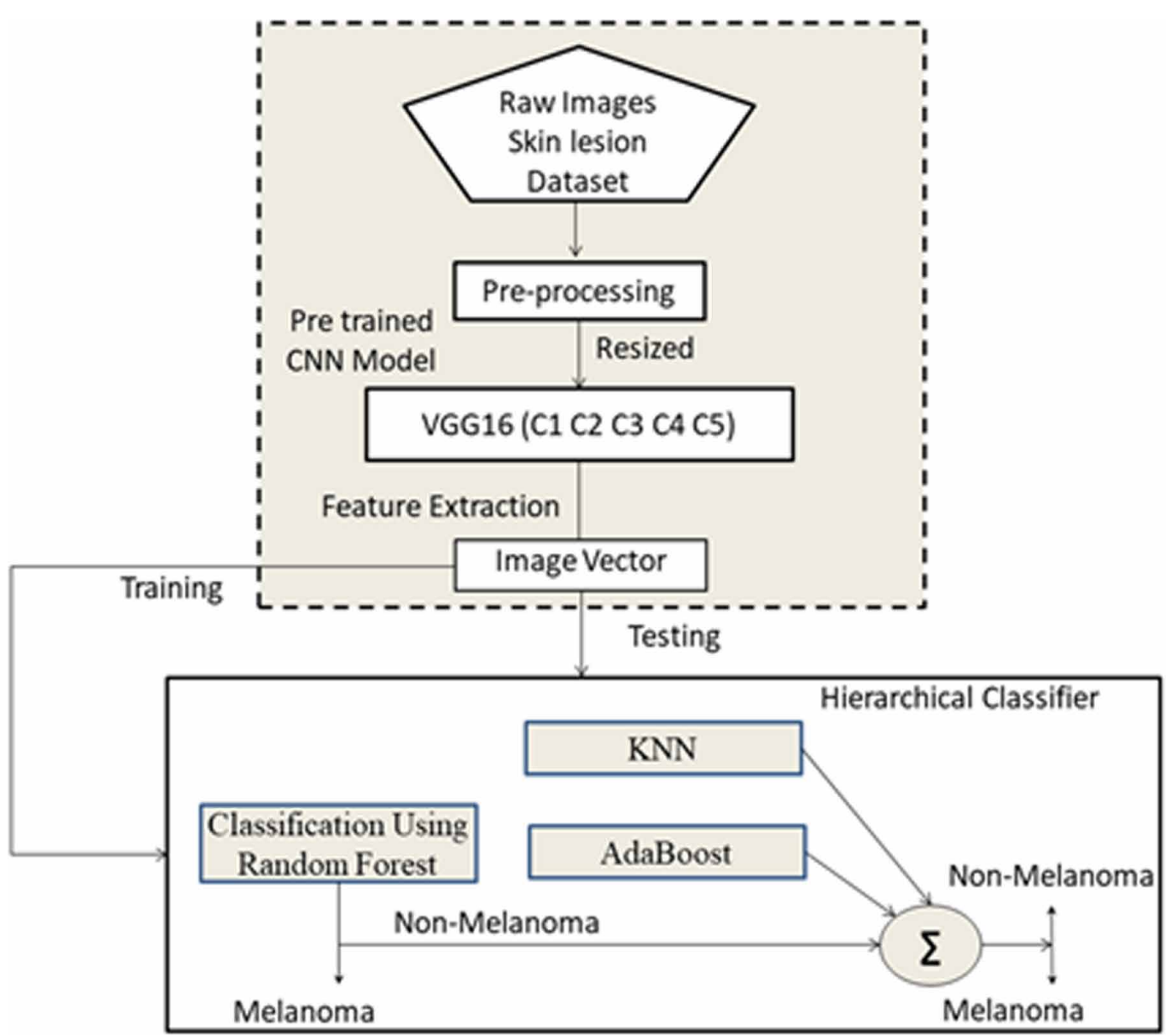

ensemble of classifiers. In majority voting, instead of considering on the accuracy of the component classifiers of the ensemble, the votes of component classifiers on the class label for an instance are collected and assign the class label with highest votes as shown below:

$y_{i}=\sum_{j} w_{j} d_{j i}$ where $w_{j} \geq 0, \sum_{j} w_{j}=1$

Where, $y_{i}$ is the output class label of the ensemble, $w_{j}$ is the weight of $j^{\text {th }}$ component classifier and $d_{j i}$ is the vote of classifier $d_{j}$ for class $C_{i}$. In majority voting, $w=1 / L$, where, $L$ is the number of components is classier in the ensemble, is taken. The block diagram and pseudo-code of the proposed approach is shown in Figure 2 and Figure 3 respectively.

\section{Experiments and Results}

The aim of the proposed approach is to detect melanoma from dermoscopic images. The dermoscopic dataset has been taken from ISIC website. ISIC: Melanoma Project is an international effort to improve melanoma diagnosis so as to reduce the mortality and the number of unnecessary biopsies. 
Figure 3. Pseudo-code of the proposed approach

\section{Proposed Algorithm}

Step1: Input=Skin_lesion_dataset;

Step2: Preprocess Input;

Step 3: Perform feature extraction using pertained VGG16 and create image vector;

Step 4: TrainedRandom_Forest, KNN andAdaBoost;

Step 5: Perform classification using Random_Forest;

Step 6: $\quad$ if (Melanoma)

\{

Step 7:

STOP;

\}

Step 8:

if (Non-Melanoma)

Step 9:

Classify using ensemble of Random_Forest, KNN and AdaBoost;

\}

\section{Step 10: Assign label using decision integration rule;}

Many international societies such as International Society for Digital Imaging of the Skin (ISDIS) and International Dermoscopy Society (IDS) are also involved in this effort. Currently, more than 13,000 dermoscopic images can be found in the ISIC archive. These images have been collected from leading clinical centers internationally and are obtained from a variety of devices within each center. Initial data sets are derived primarily from the United States, and the ISIC has ongoing commitments and contributions of additional data sets from international collaborators. The images coming to the ISIC Archive are screened for both privacy and quality assurance. Most of the images also have associated clinical metadata. The associated clinical metadata has been inspected by recognized melanoma experts. Annotation and mark-up have been done on a subset of images by recognized skin cancer experts. These mark-ups include dermoscopic features such as global and local morphological elements in the image known to discriminate between the types of skin lesions.

The dataset used in the work presented in this paper is the ISIC 2017 skin lesion classification challenge dataset. The training dataset consists of 2000 images (374 melanomas, 254 seborrheic keratoses, and 1,372 benign nevi), validation dataset consists of 150 images and the testing dataset consists of 600 images. Some of the dermocsopic images from ISIC dataset are shown in Figure 4.

The training dataset is highly imbalanced as it contains only 374 melanoma cases (around 20\%). The skewed data may produce unsatisfactory classifier. To avoid this problem, the training dataset is resampled. Various data augmentation techniques like rotation by $90^{\circ}$, rotation by $180^{\circ}$, rotation by $270^{\circ}$ and flip upside down etc. are used to balance the training dataset. As the images are of different size, pre-processing is applied and the images are resized to $224 \times 224 \times 3$, which are given as input to VGG-16. To evaluate the performance of the proposed hierarchical classifier approach, 
Figure 4. Sample of dermoscopic images from ISIC 2017 skin lesion classification challenge dataset
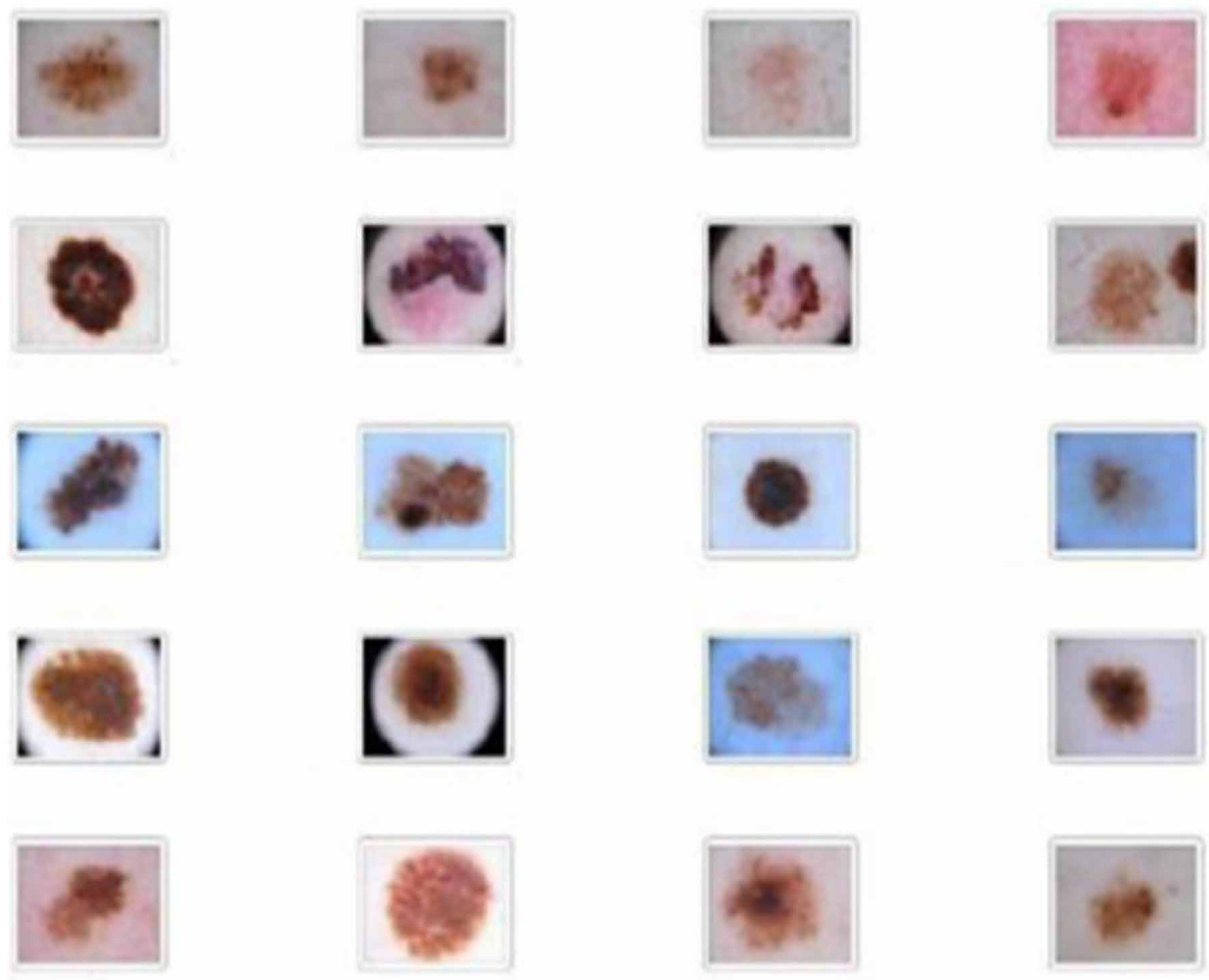

the features extracted using VGG-16 are classified using another hierarchical classifier consisting of SVM and KNN. During the experimentation, different values of ' $\mathrm{K}$ ' are taken for KNN as it affects the performance. Three performance matrices namely sensitivity, specificity and accuracy are used to evaluate and compare the classifiers. Sensitivity is the measure of true positive rate that displays the ability of a classifier in identifying the disease correctly and is calculated as:

Sensitivity $=\frac{T P}{T P+F N}$

Specificity is the measure of true negative rate that displays the classifier's ability to identify normal skin lesions and is calculated as:

Specificity $=\frac{T N}{T N+F P}$

Accuracy displays the ability of a classifier to identify the skin lesion correctly (both melanoma and non-melanoma) and is calculated as: 


$$
C A=\frac{T P+T N}{T P+F P+T N+F N}
$$

Where, TP - True positive, TN - True negative, FP - False Positive and FN - False Negative. The performance of classifier $1(\mathrm{SVM}+\mathrm{KNN})$ and classifier $2(\mathrm{RF}+\mathrm{KNN}+$ Adaboost $)$ on different values of $\mathrm{k}$ are shown in Table 1, Table 2 and Table 3. The confusion matrix of classifier 1 and classifier 2 when $\mathrm{k}=1,3$ and 5 is shown in Figure 5. It is clear from Table 1 that the proposed classifier performs better as compared to the classifier built using SVM and KNN. Also, as the value of $\mathrm{k}$ is increased, the accuracy of both the classifiers decrease.

\section{CONCLUSION AND FUTURE WORK}

In this paper, a model is proposed to detect melanoma from dermoscopic images using features extracted from VGG-16, a pre-trained deep CNN and classification is done using hierarchical classifier. The hierarchical classifier consists of RF, KNN and Adaboost. Majority voting algorithm is used to construct an ensemble of classifiers. The performance of the classifiers was evaluated on

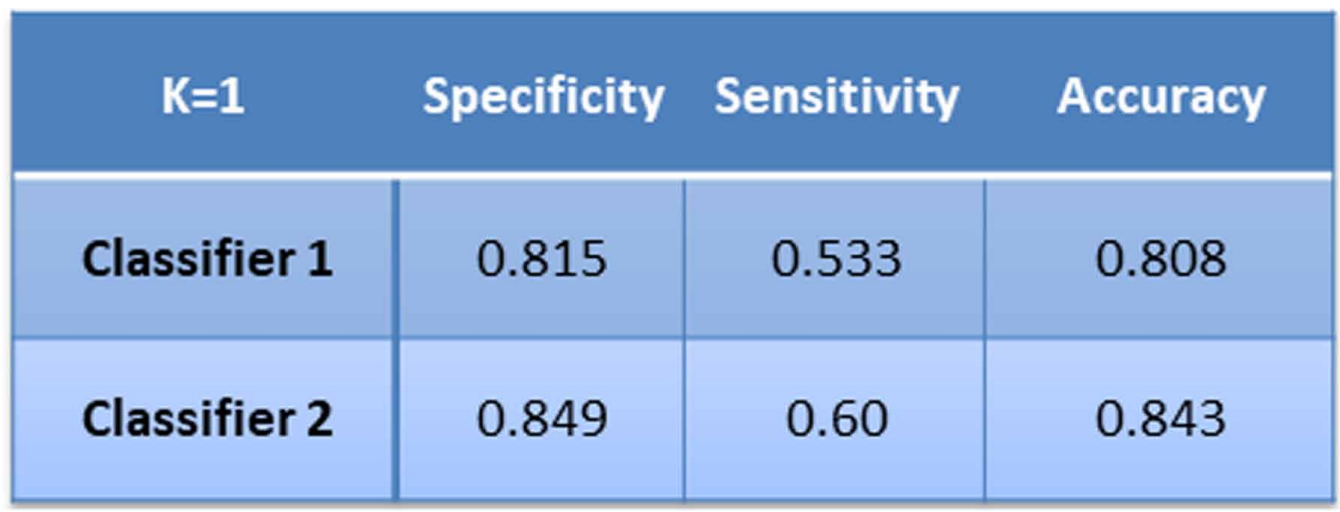

\begin{tabular}{|c|c|c|c|}
\hline$K=3$ & Specificity & Sensitivity & Accuracy \\
\hline Classifier 1 & 0.80 & 0.4 & 0.792 \\
\hline Classifier 2 & 0.829 & 0.46 & 0.82 \\
\hline
\end{tabular}




\begin{tabular}{|c|c|c|c|}
\hline$K=5$ & Specificity & Sensitivity & Accuracy \\
\hline Classifier 1 & 0.792 & 0.4 & 0.782 \\
\hline Classifier 2 & 0.819 & 0.533 & 0.813 \\
\hline
\end{tabular}

Figure 5. Confusion matrix of classifier 1 and classifier 2 at $k=1, k=3$ and $k=5$

\begin{tabular}{|c|c|c|c|c|c|c|}
\hline \multicolumn{7}{|c|}{$\mathrm{K}=1$} \\
\hline Classifier 1 & Melanoma & $\begin{array}{c}\text { Non- } \\
\text { Melanoma }\end{array}$ & & Classifier 2 & Melanoma & $\begin{array}{c}\text { Non- } \\
\text { Melanoma }\end{array}$ \\
\hline Melanoma & 8 & 7 & & Melanoma & 9 & 6 \\
\hline $\begin{array}{c}\text { Non- } \\
\text { Melanoma }\end{array}$ & 108 & 477 & & $\begin{array}{c}\text { Non- } \\
\text { Melanoma }\end{array}$ & 88 & 497 \\
\hline \multicolumn{7}{|c|}{$K=3$} \\
\hline Classifier 1 & Melanoma & $\begin{array}{c}\text { Non- } \\
\text { Melanoma }\end{array}$ & & Classifier 2 & Melanoma & $\begin{array}{c}\text { Non- } \\
\text { Melanoma }\end{array}$ \\
\hline Melanoma & 6 & 9 & & Melanoma & 7 & 8 \\
\hline $\begin{array}{c}\text { Non- } \\
\text { Melanoma }\end{array}$ & 116 & 469 & & $\begin{array}{c}\text { Non- } \\
\text { Melanoma }\end{array}$ & 100 & 485 \\
\hline \multicolumn{7}{|c|}{$K=5$} \\
\hline Classifier 1 & Melanoma & $\begin{array}{c}\text { Non- } \\
\text { Melanoma }\end{array}$ & & Classifier 2 & Melanoma & $\begin{array}{c}\text { Non- } \\
\text { Melanoma }\end{array}$ \\
\hline Melanoma & 6 & 9 & & Melanoma & 8 & 7 \\
\hline $\begin{array}{c}\text { Non- } \\
\text { Melanoma }\end{array}$ & 122 & 463 & & $\begin{array}{c}\text { Non- } \\
\text { Melanoma }\end{array}$ & 106 & 479 \\
\hline
\end{tabular}


three performance matrices namely sensitivity, specificity and accuracy. The main driving factor behind the use of hierarchal classifier is to improve the sensitivity of the system to detect melanoma correctly. The proposed hierarchical classifier performs better than classifier consist of SVM and $\mathrm{KNN}$. It can further be concluded that as the value of $\mathrm{k}$ increases, the performance of the classifier decreases. The main limitation of the work presented in this paper is that the features extracted by $\mathrm{CNN}$ are directly used to train the classifier and no feature selection is performed which is important to reduce the complexity and training time of the classifier. Another limitation of the work presented in this paper is the use of binary classifier instead of multi-class classifier.

In future, we plan to construct a multi-class classifier to classify different type of skin cancers. We also plan to perform feature selection on the extracted features using meta-heuristic techniques, in order to further enhance the classification accuracy of the proposed approach and to reduce the complexity of the classifier. 


\section{REFERENCES}

Anas, M., Gupta, R. K., \& Ahmad, S. (2017). Skin Cancer Classification Using K-Means Clustering. International Journal of Technical Research and Applications, 5(1), 62-65.

Barata, C., Ruela, M., Francisco, M., Mendonca, T., \& Marques, J. S. (2014). Two Systems for the Detection of Melanomas in Dermoscopy Images Using Texture and Color Features. IEEE Systems Journal, 8(3), 965-979. doi:10.1109/JSYST.2013.2271540

Bisla, D., Choromanska, A., Berman, R. S., Stein, J. A., \& Polsky, D. (2019). Towards Automated Melanoma Detection with Deep Learning: Data Puriðcation and Augmentation. arXiv:1902.06061v2

Brinker, T. I., Hekler, A., Enk, A. H., Berking, C., Haferkamp, S., Hauschild, A., Weichenthal, M., Klode, J., Schadendorf, D., Holland-Letz, T., von Kalle, C., Fröhling, S., Schilling, B., \& Utikal, J. S. (2019b). Deep learning Deep neural networks are superior to dermatologists in melanoma image classification. European Journal of Cancer, 119, 11-17. doi:10.1016/j.ejca.2019.05.023 PMID:31401469

Brinker, T. I., \& Klode, I. (2019a). Deep learning outperformed 136 of 157 dermatologists in a head-to-head dermoscopic melanoma image classification task. European Journal of Cancer, 113, 47-54. doi:10.1016/j. ejca.2019.04.001 PMID:30981091

Celebi, M. E., Kingravi, H. A., Uddin, B., Iyatomi, H., Aslandogan, Y., Stoecker, W., \& Moss, R. (2007). A methodological approach to the classification of dermoscopy images. Science Direct. Computerized Medical Imaging and Graphics, 31(6), 362-373. doi:10.1016/j.compmedimag.2007.01.003 PMID:17387001

Choudhury, D., Naug, A., \& Ghosh, S. (2015). Texture and color feature based WLS framework aided skin cancer classification using MSVM and ELM. 2015 Annual IEEE India Conference (INDICON), 1-6. doi:10.1109/ INDICON.2015.7443780

Dhal, K. G., Ray, S., Sen, M., \& Das, S. (2018). Proper Enhancement and Segmentation of the Overexposed Color Skin Cancer Image. In Intelligent Multidimensional Data and Image Processing. IGI Global.

Dorj, U. O., Lee, K. K., Choi, J. Y., \& Lee, M. (2018). The skin cancer classification using deep convolutional neural network. Multimedia Tools and Applications, 77(8), 9909-9924. doi:10.1007/s11042-018-5714-1

Dubai, P., Bhatt, S., Joglekar, C., \& Patil, S. (2017). Skin cancer detection and classification. Electrical Engineering and Informatics (ICEEI), 6th International Conference on IEEE.

Esteva, A., Kuprel, B., Novoa, R., Ko, J., Swetter, S., Blau, H., \& Thrun, S. (2017). Dermatologist-level classification of skin cancer with deep neural networks. Nature, 542(7639), 115-118. doi:10.1038/nature21056 PMID:28117445

Firmansyah, H. R., Kusumaningtyas, E. M., \& Hardiansyah, F. F. (2017). Detection melanoma cancer using $\mathrm{ABCD}$ rule based on mobile device. Knowledge Creation and Intelligent Computing (IES-KCIC), International Electronics Symposium on IEEE.

Giotis, I., Molders, N., Land, S., Biehl, M., Jonkman, M. F., \& Petkov, N. (2015). MED-NODE: A computer assisted melanoma diagnosis system using non-dermoscopic images. Expert Systems with Applications, 42(19), 6578-6585. doi:10.1016/j.eswa.2015.04.034

Gopalakrishnan, K., Khaitan, S. K., Choudhary, A., \& Agrawal, A. (2017). Deep Convolutional Neural Networks with transfer learning for computer vision-based data-driven pavement distress detection. Construction \& Building Materials, 157, 322-330. doi:10.1016/j.conbuildmat.2017.09.110

Havaei, M., Davy, A., Warde-Farley, D., Biard, A., Courville, A., Bengio, Y., Pal, C., Jodoin, P., \& Larochelle, H. (2016). Brain tumor segmentation with deep neural networks. Medical Image Analysis, 35, 18-31. doi:10.1016/j. media.2016.05.004 PMID:27310171

Jafari, M. H., Nasr-Esfahani, E., Karimi, N., Soroushmehr, S. M. R., Samavi, S., \& Najarian, K. (2017a). Extraction of skin lesions from non-dermoscopic images using deep learning. International Journal of Computer Assisted Radiology and Surgery, 12, 1021-1030. doi:10.1007/s11548-017-1567-8 PMID:28342106 
Jafari, M. H., Samavi, S., Soroushmehr, S. M. R., Mohaghegh, H., Karimi, N., \& Najarian, K. (2017b). Set of Descriptors for Skin Cancer Diagnosis Using Non-Dermoscopic Color Images. IEEE International Conference on Image Processing (ICIP).

Jain, S., Jagtap, V., \& Pise, N. (2015). Computer aided melanoma skin Cancer detection using image processing. Procedia Computer Science, 48, 736-741. doi:10.1016/j.procs.2015.04.209

Kalouche, S. (2016). Vision-Based Classification of Skin Cancer using Deep Learning. Academic Press.

Kavitha, J. C., Suruliandi, A., \& Nagarajan, D. (2017). Melanoma Detection in Dermoscopic Images using Global and Local Feature Extraction. International Journal of Multimedia and Ubiquitous Engineering, 12(5), 19-28. doi:10.14257/ijmue.2017.12.5.02

Krizhevsky, A., Sutskever, I., \& Hinton, G. (2012). ImageNet Classiðcation with Deep Convolutional Neural Networks. Advances in Neural Information Processing Systems.

Li, Y., \& Shen, L. (2018). Skin Lesion Analysis Towards Melanoma Detection Using Deep Learning Network. Sensors (Basel), 18(2), 556. doi:10.3390/s18020556 PMID:29439500

Melinscak, M., Prentasic, P., \& Loncaric, S. (2015). Retinal vessel segmentation using deep neural networks. Proceedings of International Conference on Computer Vision Theory and Applications, 557-582. doi: $10.5220 / 0005313005770582$

Mhaske, H. R., \& Phalke, D. A. (2013). Melanoma skin cancer detection and classification based on supervised and unsupervised learning. International conference on Circuits, Controls and Communications (CCUBE), 1-5. doi:10.1109/CCUBE.2013.6718539

Priyanga, P., \& Naveen, N.C. (2018). Analysis of Machine Learning Algorithms in Health Care to Predict Heart Disease. International Journal of Healthcare Information Systems and Informatics, 13(4), 82-97.

Puyalnithi, T. \& Vankadara, M. (2018). A Unified Feature Selection Model for High Dimensional Clinical Data Using Mutated Binary Particle Swarm Optimization and Genetic Algorithm. International Journal of Healthcare Information Systems and Informatics, 13(4), 1-14.

Salido, J. A. A., \& Jr. Ruiz, C. (2018). Using Deep Learning to Detect Melanoma in Dermoscopy Images. International Journal of Machine Learning and Computing, 8(1), 61-68. doi:10.18178/ijmlc.2018.8.1.664

Seetharaman, K. (2019). Melanoma Image Classification Based on Multivariate Parametric Statistical Tests of Hypothesis. In Advanced Classification Techniques for Healthcare Analysis. IGI Global.

Simonyan, K., \& Zisserman, A. (2014). Very Deep Convolutional Networks for Large-Scale Image Recognition. Proceeding of International Conference on Learning Representations. https://arxiv.org/abs/1409.1556

Szegedy, C., Liu, W., Jia, Y., Sermanet, P., Reed, S., Anguelov, D., Erhan, D., Vanhoucke, V., \& Rabinovich, A. (2015). Going deeper with convolutions. In Proceedings of the IEEE Conference on Computer Vision and Pattern Recognition. The International Skin Imaging Collaboration. Available from https://isic-archive.com

Priti Bansal is Assistant Professor in Department of IT, NSUT, Delhi, India. She received her Ph.D in computer engineering from University of Delhi. Her area of interest includes software engineering, machine learning, soft computing, and metaheuristic algorithms. 